\title{
SARS-Cov2: A Review of this Novel Coronavirus
}

\author{
Dominic Worku' ${ }^{*}$ and Elliott Worku \\ ${ }^{1}$ Internal Medicine Trainee, St Georges Hospital, London, United Kingdom \\ ${ }^{2}$ ICU fellow, Adult Intensive Care Services, The Prince Charles Hospital, Chermside, Brisbane, QLD Australia
}

${ }^{\star}$ Corresponding Author: Dominic Worku, Internal Medicine Trainee, St Georges Hospital, London, United Kingdom; E-Mail: dominicworku@hotmail.co.uk

Received: March 24, 2020; Accepted: March 26, 2020; Published: April 03, 2020

\begin{abstract}
Background: Coronaviruses are a group of RNA viruses responsible for respiratory and gastrointestinal illness. Of this group two have been associated with pandemics in recent years. As of December 2019, a new coronavirus (SARS-CoV2) was reported in Wuhan, China which despite best efforts has spread internationally prompting panic and universal public-health measures.
\end{abstract}

Methods: A literature review was conducted utilising the PubMed database in March 2020. Search terms included 'Wuhan' OR 'Coronavirus' OR '2019nCoV' OR 'SARS-CoV2' AND 'pneumonia' OR 'Outbreak' OR 'Infection'. These were used in isolation and combination to yield results. Papers were selected if they explored the diagnosis, management, transmission and/or treatment of SARS-CoV2. Following these 68 papers were screened of which 48 papers were included in this review.

Results \& Discussion: Early studies highlight that SARS-CoV2 has an incubation period of between 3-7 days and commonly presents with fever $(93 \%)$, cough $(69.8 \%)$ and dyspnoea $(34.5 \%)$ and prominent upper respiratory tract symptoms. Patients are predominantly male and there is high prevalence of significant comorbid disease in fatal cases (overall case: fatality ratio-2-3\%). Encouragingly, supportive treatments are mainstay with the need for invasive ventilation and extracorporeal life support (ECLS) low. Diagnosis of SARS-CoV2 is made by demonstration of the virus by RT-PCR of throat/lavage specimens; however given the propensity for false negatives, CT-imaging is being used diagnostically with characteristic findings reported and can even detect disease in the asymptomatic phase where transmission is possible. International interventions have been to adhere to 14-day observation periods for suspected cases, the wearing of N95 facemasks alongside social distancing and hand-hygiene due to fomites. While antiviral treatments have been trialled in case-series no clear consensus has been made regarding their use but it remains clear that concurrent antibiotics are mainstay with restrictive fluid replacement. Emerging therapies which may show benefit include chloroquine, remdesivir, tocilizumab, azithromycin and sunitinib with the discontinuation of ACE inhibitors proposed. Conclusion: SARS-CoV2's impact is greatest in the elderly and comorbid. Research has indicated key targets which may be important in producing effective treatments and an efficacious vaccine. On-going aims must be to try to alter behaviours and limit viral spread through social distancing, good hand hygiene and Personal Protective Equipment (PPE). Future trials must attempt to prognosticate patients further and understand the role if any of redeployed treatments which have showed some promise. In addition, questions surrounding long term immunity require further investigation.

\section{Introduction}

Coronaviruses are a group of spherical positive-strand RNA viruses. Prior to SARS-CoV2 (formerly known as Ncorv-19/covid-19) six coronaviruses were thought to exist, with two causing pandemics in recent times: Severe Acute Respiratory Syndrome (SARS) and Middle Eastern Respiratory Syndrome (MERS). This group of viruses are not unique to humans and can be found in birds, domesticated animals, camels and bats who serve as hosts and reservoirs of the disease $[1,2]$. It is thought that $30 \%$ of annual respiratory illnesses are caused by coronaviruses leading to wide ranging presentations from pharyngitis to pneumonia, with a predominantly respiratory droplet spread. Increasingly, human-human transmission and transmission by asymptomatic carriers of coronaviruses is being appreciated $[3,4]$.

On $8-9^{\text {th }}$ December 2019,7 patients presented within Wuhan, Hubei province, China with a viral pneumonia of unknown origin, but a common exposure to the Huanan seafood market where many wild animals are held in close proximity $[1,5]$. As of $31^{\text {st }}$ December the World Health Organization (WHO) were informed of these novel cases and the first identification of this virus as a coronavirus was on the $9^{\text {th }}$ January 2020. Complete analysis of the viral genome revealed it is a beta coronavirus with $79 \%$ similarity to SARS and $50 \%$ similarity to MERS, which are also beta coronaviruses, but is nearly completely homologous with two bat derived SARS who are the likely host and reservoir of the SARS-CoV2. The viral genome was made publicly available from the $12^{\text {th }}$ January 2020 allowing for the first Reverse Transcriptase polymerase chain reaction (RT-PCR) testing to occur $[3,6]$. The structure of a coronavirus is such that there are Spike (S), Envelop (E) and membrane (M) proteins. The (S) protein is the primary determinant of cellular infection and it itself has two domains; S1 which mediates receptor binding, and S2 which allows for virion fusion to the cell membrane. Distinct in SARS-CoV2 is an additional activation loop in the S1/S2 proteins not shared with either MERS or SARS [7]. As of $23^{\text {rd }}$ March 2020, 170 countries have declared cases, with patient numbers globally standing at $>300,000$, and over 14,509 deaths with a reported case fatality rate of $2-3 \%$ which is much lower than initial reports highlighted $[8,9]$. In comparison MERS spread to 27 countries and resulted in 858 deaths with a case fatality rate of $34.4 \%$ 
with SARS affecting 26 countries with 774 deaths: a corresponding case fatality rate of $9.5 \%$. It is because of the inexorable spread of this virus that as of $30^{\text {th }}$ January 2020 the WHO branded SARS-Cov2 as a global public emergency. This has resulted in a multitude of countries taking enormous steps to limit international travel, and more dramatic interventions here in the UK for example with public transport limited to key workers. This was provoked by by the first wave of mortality here in the UK and quarantine in Italy $[10,11]$. Borne from this panic there are increasing reports of discrimination against Chinese people, and mounting hysteria with regards to the possible apocalyptic nature of this outbreak. However, even with the rapid increase in cases noted through means such as a contact tracing and screening, overall case: fatality rate remains $2 \%$ with severe disease occurring predominantly in the elderly with attendant comorbidities [12].

In this review we explore the knowns of SARS-Cov2 regarding its prevention, diagnosis, presentation and management and the vast unknowns which have made this disease entity the one of the most globally important of the century so far.

\section{Methods}

Literature review was conducted in March 2020. Papers were identified via a literature search using the PubMed database. Papers were selected if they were written in English and published in peer reviewed journals between December and March 2020. Articles were selected if they documented cases of SARS-CoV2 (formerly known as $2019-\mathrm{nCoV} / \mathrm{n}-\operatorname{cov} 19)$ and dealt with the diagnosis, management, transmission and/or treatment of the infection.

Search terms included 'Wuhan' OR 'Coronavirus' OR '2019-nCoV' OR 'n-cov19' OR 'SARS-CoV2' AND 'pnuemonia' OR 'Outbreak' OR 'Infection'. These terms were used in isolation and combination to locate search results.

This search obtained 78 results from which both authors conducted a screen of their titles, study design and abstracts. Issues highlighted at this stage were addressed by contacting the paper authors. Papers were excluded if they were not written in English, not peer reviewed, did not explore in detail the diagnosis, management, transmission and treatment of SARS-CoV2 leading to 57 papers. Data was extracted from each paper regarding the population, end points, follow up and methods employed. All papers with a conflict of interest or, publication bias and overlapping patient or outcome data were removed. Application of the above criteria yielded 48 papers which were utilised in this review.

\section{Results and Discussion}

Following the identification of SARS-CoV2, much has been done to understand the natural history of the condition and its presentation. Early studies revealed that SARS-CoV2 has an incubation period of between 3-7 days with a case series reporting a median of 7 days (4.0-8.0 days) between symptom onset and hospitalisation and from symptom onset to ICU admission 10.5 days (8.0-17.0 days) [5, 13]. In terms of presentation fever (93\%) was the most common initial symptom followed by cough $(69.8 \%)$, dyspnoea $(34.5 \%)$, sore throat (15\%), headache $(7.2 \%)$ and diarrhoea $(6.1 \%)$ which corroborates with other studies $[5,6]$. These symptoms are common amongst the betacoronaviruses however SARS-CoV2 uniquely also presents with upper respiratory tract infection (URTI) symptoms including sore throat, rhinorrhoea, anosmia and sneezing. Of those patients who went on to die, death occurred between 6-41 days after symptom onset (median 14 days). Unsurprisingly in those patients $>70$ years old the interval between symptom and onset and death was significantly decreased $[13,15]$.

To date one of the largest reviews of SARS-CoV2 patients $(n=278)$ utilised data from 3 large scale studies. Of included patients $61.9 \%$ were male with an average age of 54.7 years. This gender disparity is described elsewhere in the literature with a male preponderance of up to $70 \%$ reported [16]. Among study patients the most common comorbid conditions included hypertension (15-31\%), cardiovascular disease (14-40\%) and type 2 diabetes mellitus (10-19\%). Results from this review revealed that 72 patients required Intensive Care Unit (ICU) admission, 56 of these being diagnosed with Acute Respiratory Distress Syndrome (ARDS). Of these 23 ( $8 \%$ total cohort) required invasive ventilations and 9 (3\% total cohort) required extracorporeal life support (ECLS). Overall mortality differed amongst the incorporated studies ranging from $4-14 \%$ with inherent differences between study groups [6]. In a review of 99 patients with SARS-CoV2 treated at a major tertiary infectious disease hospital in Wuhan, only $4 \%$ required invasive ventilation and $3 \%$ required ECLS respectively. Importantly these patients could be predicted to need higher levels of support with existing early warning score models. However, no comparison of patients in these studies receiving such treatments in the context of their MuLBSTA score was undertaken. The MuLBSTA score is a validated tool for assessing the 90-day mortality risk using 6 parameters in the setting of viral pneumonia, somewhat synonymous with the CURB-65 severity score of community acquired pneumonias $[17,18]$. Formal studies are required to assess the use of this scoring system and others in predicting outcomes in SARS-CoV2.

Common amongst these multiple reviews is the higher prevalence of disease in middle-aged men with pre-existing cardiovascular disease. One debated risk factor is that of smoking. Interestingly, smoking status in affected patients is both poorly recorded and investigated. Of the one systematic review that could be found on this subject utilizing only 5 papers, a positive association between smoking and both disease progression (OR 1.28) and ICU admission (RR 2.4) was recorded. However no relationship between relative tobacco exposures or e-cigarette use and these outcomes was explored $[8,19]$. While larger and further studies are needed it would seem prudent that physicians and health agencies reinforce the need for smoking cessation especially as the public are possibly more motivated to follow health messages at this time. While it is necessary in a minority to utilise invasive ventilation and ECLS, it remains unclear what benefit the latter will offer in SARS-CoV2. Whilst ECLS was utilised in SARS to modest effect, mortality reduction was seen in some cohort studies of MERS [20,3]. However, experience from the larger H1N1 pandemic of 2009-2011 has cast doubt regarding the efficacy of ECLS in ARDS of viral aetiology, with the EOLIA trial suggesting that 60 -day mortality is not altered with this therapy versus invasive ventilation. Recent guidance from the WHO recommends prone 
ventilation, restrictive fluid practice and lung protective ventilation. Whilst there has been enthusiasm for corticosteroid use in SARSCoV2 ARDS, there is little evidence for their prescription, and they are not currently recommended having shown no promise in SARS or MERS previously. At present high-flow nasal oxygen therapy is considered a good highly available intervention that can in patients prevent the need for intubation but requires intense monitoring [21-25]. Given the large numbers of patients affected by SARS-CoV2, massive coordinated action must be undertaken by health agencies to increase both ICU beds and expertise amongst non-ICU trained staff on traditional hospital wards. It may be however that the propensity for severe respiratory disease in some patients may be linked to neurological involvement. Evidence from studies of SARS in both human and animal models indicated it could cause severe brainstem infection and by involving the cardiorespiratory centres depress respiration centrally. Whilst this is difficult to reverse, awareness of this possible mechanism of respiratory decline is important for all physicians to consider in the nonresponding patient [26].

As mentioned previously, predicting outcomes in patients is key to allow for health services to deploy resources accordingly. Analysis of patients requiring ICU admission versus those who did not revealed statistically increased total neutrophils, decreased lymphocyte counts and increased serum pro-inflammatory cytokines levels (e.g. IL-6, $\mathrm{TNF} \alpha$ ), highlighting the role of immune activation and the cytokine storm' in SARS-CoV2 infection. Moreover, serum IL-6 and TNFa levels have been found to correlate with risk of death in SARS-CoV2 infection $[16,8]$. Key to the innate immune systems response to viral infection is the production of interferon following recognition of viral associated pathogen associated molecular patterns (PAMPs) such as viral RNA by toll-like-receptors leading to activation of signalling cascades. It appears clear that there is attenuation of this process in the closely related SARS infection, with immune evasion mechanisms at work including degradation of RNA detecting proteins, which in part explains the prolonged incubation period of SARS-CoV2 versus other common viral infections. For long term control of viral infections Th1 adaptive immune responses are needed with evidence $\mathrm{Th} 2$ responses in SARS-CoV2 is linked to negative outcomes. Therefore, a prerequisite for any future vaccines trialled in SARS-CoV2 must be to produce this response with DNA based vaccines more likely to elicit strong durable immune responses of this nature [27].

Currently the diagnosis of SARS-CoV2 requires pathological evidence of the virus through RT-PCR of suitable samples (e.g throat swab/bronchoalveolar lavage (BAL), however radiological imaging is important in determining both severity of disease and ruling out differentials [13]. Imaging studies (CXR/Computer Tomography (CT)) of affected patients demonstrates that SARS-CoV2 presents variably with bilateral ground glass opacifications the most common finding, but focal/lobar consolidation, pleural effusion, mediastinal lymphadenopathy and pneumothorax also being described $[4,28]$. In addition CT findings vary in SARS-CoV2 infection depending on the underlying stage of the disease (Table 1). Currently no serological testing is routinely used in SARS-CoV2 diagnosis.

One study assessing the range of radiological findings of SARSCoV2 in 90 patients (39 men; 51 women; median age 50 years) showed that on baseline noncontrast CT 69/90 (77\%) had radiological abnormalities with 53\% having multiple lobe involvement with a predilection for the lower lung lobes noted. The most common radiological pattern was ground glass opacification (72\%) followed by consolidation (13\%), in addition $56 \%$ had pleural thickening. It is thought ground glass opacities represent diffuse alveolar damage and their filling with pus or exudate. Of the 52 patients reimaged between 1-6 days post initial CT, 19\% had no changes, $73 \%$ had disease progression and $3 \%$ demonstrared new bilateral ground glass opacities having had normal previous imaging [28]. Interestingly, in a retrospective analysis of 51 confirmed patients with SARS-CoV2 of median age 45 years, while only 36 (71\%) had positive RT-PCR throat swabs on first testing with a further 12 testing positive on the second throat swab this compared to 50/51 (98\%) on initial noncontrast CT showing signs of viral pneumonia $(\mathrm{p}<0.001)$ [29]. This corresponds to a large review of 1014 cases in China where the sensitivity of chest CT was $97 \%$, with $70 \%$ of patients with a negative RT-PCR test showing typical CT findings for SARS-Cov2 infection. This highlights an important fact that the total positive rate of RT-PCR of throat swabs is between $30-60 \%$ and as such cannot be relied upon by itself for diagnosis and may miss affected patients. Importantly, the time between initial negative to positive RT-PCR testing of throat swabs can be up to 6 days [30]. Moreover, there have been findings of CT changes in keeping with SARS-CoV2 in otherwise asymptomatic patients suggesting that even subclinical disease can lead to pathological changes to the lung space and therefore a low threshold of imaging should be used in patients with suitable exposure to allow for earlier treatment [4]. Therefore, the ongoing focus for diagnosis must be to further optimise the RT-PCR testing process including sample collection, transport and storage to ensure no possible tampering of results. Clinicians must therefore not be deterred by a negative result in the context of a high index of suspicion; although vigilance for superadded bacterial or fungal processes should be both considered and investigated as required.

Table 1: CT findings by stage of SARS-CoV2 infection [13].

\begin{tabular}{|c|ll|}
\hline Stage (Days) & \multicolumn{1}{c|}{ CT Findings } \\
\hline Ultra-Early & • Focal / ground glass nodules \\
\hline Early [1-3] & • & $\begin{array}{l}\text { Congestion of alveolar capillaries, interstitial } \\
\text { oedema }\end{array}$ \\
\hline Rapid Progression [3-7] & • & Worsening alveolar and interstitial oedema \\
\hline Consolidation [7-14] & $\bullet$ & $\begin{array}{l}\text { Multiple patchy consolidation, fibrous alveolar } \\
\text { exudates }\end{array}$ \\
\hline Dissipation [14-21] & $\bullet$ & Strip like consolidation \\
\hline
\end{tabular}

At present no cases of death have been reported in children, although cases have been reported in children between ages 1.5 months-17 years and appear to manifest in a similar way to adult patients albeit milder with recovery anticipated within 1-2 weeks [31]. It remains unclear the possible mechanism of this protection afforded by youth. Encouragingly there is no evidence of maternal 
transmission of SARS-CoV2 during pregnancy like both SARS and MERS. However, it is in this patient group that worse outcomes are seen with pneumonia of all types with $25 \%$ of those affected needing ventilatory support [1]. While no formal cases of SARS-CoV2 have been identified in pregnant women lessons can be learnt from the SARS and MERS pandemic. In the seven cases of SARS in pregnant women in Hong Kong: four sustained spontaneous miscarriage in the first trimester. Moreover, in case control studies pregnant women with SARS were more likely to suffer renal failure and disseminated intravascular coagulopathy ( $\mathrm{p}=0.006$ ) with $60 \%$ and $40 \%$ requiring ICU admission and intubation respectively. In MERS of the five documented cases all required ICU, with two mothers dying, and two cases of perinatal death recorded [1]. As such a low threshold for testing and isolating pregnant women is needed, and while no link to the unborn child has been found given the severity of related coronaviruses in this patient group, treatment should be given without delay.

Key to the control of this pandemic is to limit the spread of SARS$\mathrm{CoV} 2$ which is linked to the transmissibility of the virus itself. One key aspect is its reproductive rate ( $\mathrm{R} 0$ value). This refers to the average number of people who will be infected from one contagious person and is quoted to allow us to predict potential spread of a pathogen. Based on initial data and cases as they appeared, quoted R0 values have included 1.68 (31 December - Jan 2020) 2.24 (10-24 January 2020) [6], case reports quoting 5.5 with the WHO quoting between 1.4-2.5. This is in keeping with most major viral infections which have resulted in global concern (Table 2) [32,33]. It remains to be seen if the R0 of SARs-CoV2 continues to change, this is because notable among the coronaviruses is the frequency of both replication errors and recombination events that can take place during its reproductive cycle and can result in quasispecies with increased virulence. So far analysis of the SARS-CoV2 genome shows little evidence of significant recombination. However, with more cases and geographical spread of the virus this may change [33]. One way to decrease the R0 has been to limit both domestic and international travel with at international airports and borders thermal scanning being undertaken to detect possible cases however when this method was evaluated during the H1N1 pandemic a sensitivity of only $5.8 \%$ was reported. Regardless however it can be considered important in raising public awareness of the condition and improve overall participation in risk reducing behaviours such as social distancing [2]. Upon analysing the effect of travel restrictions on SARS-CoV2 dissemination since $23^{\text {rd }}$ January 2020, utilising the Global Epidemic and Mobility Model (GLEAM), it has been shown that even in the instance of $90 \%$ travel reductions if the transmission rate of SARS-CoV2 remains constant then the delay achieved in peak number of cases is only by 2 weeks with the peak number of cases in Wuhan expected in March 2020. Therefore, key to any government strategy for SARS-CoV2 is to propagate effective hygiene behaviours and enforce quarantine. This requires considerable investment in easy to access, reputable public information sources and importantly quashing of false information that can now be easily disseminated through social media services. [34] At present common practices include 14 days observation period for exposed and symptomatic persons and to attend hospital if concerned or systemically unwell, wearing of N95 masks by healthcare workers who have had close contact to affected cases and standing at least $1 \mathrm{~m}$ from other members of the public. These recommendations are supported by the Centres for Disease Control and Prevention (CDC) and have since become internationally adopted [13, 35]. Suspected cases here were defined as those individuals who have been in close contact with confirmed cases in a workplace/study/ vehicular environment or have been involved treating a confirmed case. These recommendations are even more relevant given the first reports of human-human transmission $\left(20^{\text {th }}\right.$ January 2020) which involved a family cluster with a male in Vietnam developing the infection having never travelled to an affected area but only having contact with his parents who had flown from Wuhan some 3 days earlier.[36] Moreover, in the hospital environment reports have circulated of nosocomial outbreaks of SARS-CoV2 highlighting the need for strict hand hygiene and Personal Protective Equipment (PPE) use particularly in aerosol generating procedures. One study assessing 3 patients treated in Singapore in isolation rooms with air sampling, environmental and PPE sampling showed that there was significant environmental and PPE contamination, particularly on the toilet bowel and sinks but not outside of the isolation rooms themselves. This highlights firstly the possibility of faecal shedding of the virus further suggested by the symptoms of diarrhoea in reported cases but also the awareness needed of fomite transmission Emerging evidence suggests the virus can persist on plastic and metal surfaces for up to 3 days. Interestingly after routine decontamination practices these isolation rooms showed no contamination whatsoever proving current procedures are adequate when performed properly and regularly and that adequate PPE resources are mandatory to limit hospital spread $[37,38]$. One contentious issue however is mass public masking. This is for several reasons this includes not only a global shortage of masks but also a lack of evidence regarding its effectiveness. While logically their use limits droplet spread, they have never been truly studied in this regard being designed for occupational not environmental exposures. It is because of this the WHO offers rather vague guidance in this regard. However, it must be remembered that if hand hygiene isn't followed than masks can usher a false sense of security and can easily become contaminated and thereby useless. Regardless clear alternatives are needed given that disposable surgical masks provide huge financial and environmental burdens [39].

Table 2: Relative reproductive rates of commonly encountered viruses [32, 33].

\begin{tabular}{|l|c|}
\hline \multicolumn{1}{|c|}{ Virus } & R0 value \\
\hline SARs-CoV & $2-5$ \\
\hline MERS-CoV & $<1$ \\
\hline H1N1 & $1.2-1.6$ \\
\hline Rhinovirus & 6 \\
\hline HIV & $2-4$ \\
Measles & $12-18$ \\
Influenza & $1-2$ \\
Ebola & $1.5-2.5$ \\
\hline
\end{tabular}


At present there is no consensus on the treatment of SARSCoV2. Likewise, while many treatments were trialled in the case of SARS/MERS no vaccine was produced. Review of the literature reveals that antibiotics are being trialled in up to $90 \%$ with $76-85 \%$ receiving antivirals. Other treatments trialled include intravenous immunoglobulin (IVIg) and corticosteroids but none have demonstrated dramatic success $[6,17]$. Antiviral choice so far has included among others oseltamivir, ganciclovir [17], lopinavir/ritonavir [2] and while a tendency to improved outcomes and/or reduced viral loads have been reported these have been restricted by their small sample sizes and inadequate methodology. Novel medications that may help in SARS-CoV2 infection include combination chloroquine with remdesivir and hydroxychloroquine. Indeed, in a multiple drug panel analysis looking at half-cytotoxic concentration and selectivity index both chloroquine and remdesivir (adenosine analogue) were able to do so at low micromolar concentrations in in vitro assays and have a proven safety record with the former of these used for some 70 years in clinical medicine $[6,40]$. Indeed since this initial report intravenous remdesivir has been given in a case report with a positive outcome [41]. Underlying chloroquine's antiviral effect is alteration to lysosomal $\mathrm{pH}$ which is thought may reduce SARS-CoV2 membrane binding with hydroxychloroquine demonstrating up to 3 times the potency of chloroquine in vitro. Of the few open label clinical trials that exist, one demonstrated that of the 20 patients who received hydroxychloroquine (of which 6 also received azithromycin) a reduction at 6 days in viral carriage of $57.1 \%$ and $100 \%$ was reported. No evidence of cardiotoxicity was observed in these patients. However, this study was limited by its high dropout rate and limited follow up but does demonstrate some possible means of both prophylaxis and treatment in shedding patients [42]. Common to some of these drugs are their anti-rheumatic properties and it may be that rheumatoid arthritis patients who are on these drugs are less likely to develop SARSCoV2, although this has not yet been epidemiologically demonstrated. IL-6 blockers (e.g. tocilizumab) have been among rheumatoid drugs shown to have some efficacy in SARS-CoV2 infection and can be assumed to limit the cytokine storm which has been observed in critically ill patients. Small initial retrospective studies in SARSCoV2 infection patient show that tocilizumab can produce clinical, biochemical and radiological improvements even in severe disease and has now been chosen for phase 2 trials [43]. As we understand more about SARS-CoV2 life cycle so has our identification of potential drug targets. It is now appreciated that SARS-CoV2 utilises the Angiotensin Converting Enzyme 2 (ACE2) receptor to enter mammalian cells via receptor mediated endocytosis with TMPRSS2 a serine protease implicated in this process. The ACE2 receptor is ubiquitous but is particularly expressed within AT2 alveolar epithelial cells which are prone to viral infection and raises two main points. Firstly, Animal studies suggest that ACE inhibitors can significantly increase ACE2 activity, and therefore may potentiate SARS-CoV2 virulence. As such we must determine whether we can limit the risk of severe disease by switching these agents when appropriate. This would be a very easy and inexpensive option that could be pre-emptively done [44, 45]. Secondly, key to infection of these cells is AP2-associated protein kinase 1 (AAK1), which on drug screening is effectively blocked by sunitinib and erlotinib and could represent potential drug targets. It is thought this would offer a means of decreasing virion entry into cells alongside camostate mesylate, a TMPRSS2 inhibitor which has proven in vitro activity in preventing SARS-CoV2 infection in multiple lung cell lines. However, sunitinib and others of the class have significant side-effects such as Posterior Reversible Encephalopathy Syndrome (PRES), Thyroiditis and pancytopenia. Baracitnib, a closely related JAK-kinase inhibitor may avoid these issues however safety concerns with regards to potentiating progression of SARS-CoV2 infection have been raised [44, 46, 47]. While targeting of the S1 subunit of the virus seems ideal considering its role in entering susceptible cells , previous studies in MERS have shown the (E) protein is integral and that when it is deleted both defective viral propagation and beneficial host immune responses to the virus are seen. Considering this (E)protein based therapies may possibly yield promising results. Suitable animal models could include ferrets as they can replicate some of the URTI symptoms of the condition and therefore the main method by which SARS-CoV2 spreads this, deserves further investigation $[3,48]$.

\section{Conclusion}

SARS-CoV2 represents the $7^{\text {th }}$ coronavirus and is novel in both its genomics, degree of spread and the fear it has inspired. Thus far its impact is greatest in the elderly and comorbid. Early institution of mechanical ventilation to control droplet spread may be important with consequences for critical care service planning, and in more severe ARDS presentations, there will be increasing demand for invasive therapies, which should primarily be undertaken by experienced centres with high case volume. Research has indicated key targets which may be important in producing an efficacious vaccine, but this may not be available until later waves of disease, as has been seen with prior pandemics. At present current aims must be to try to alter behaviours and limit viral spread, especially as we expect to reach the peak number of cases soon and aware of the viruses stability on surfaces. In established severe disease, lung protective ventilation and prone positioning will be important. Trials should now be undertaken to further prognosticate patients and understand the role of redeployed treatments have in SARS-CoV2 infection as well as the likelihood of possible long-term immunity in those who survive.

\section{References}

1. Shwartz DA, Graham AL (2020) Potential Maternal and infant outcomes from coronavirus 2019-nCoV (SARS-Cov-2) Infection in pregnant women: lessons from SARS, MERS and other human coronavirus. infections. Virus 12: 194. [Crossref]

2. Kim JY, Choe PG, Oh Y, Oh KJ, Kim J, et al. (2020) The first case of 2019 novel coronavirus pneumonia imported into Korea from Wuhan, china: implication for infection prevention and control measures. J Korean Med Sci 35. [Crossref]

3. Ralph R, Lew J, Zeng T, Francis M, Xue B, et al. (2020) 2019-nCoV (Wuhan virus), a novel coronavirus: human-to-human transmission, travel related cases, and vaccine readiness. The Journal of Infection in Developing Countries 14: 3-17. [Crossref]

4. Fuk-Woo Chan J, et al. (2020) A familial cluster of pneumonia associated with the 2019 novel coronavirus indicating person-to-person transmission: a study of a family cluster. Lancet.

5. Wu D, Wu T, Liu Q, Yang Z (2020) The SARS-CoV2 outbreak: what we know. International Journal of Infectious Diseases.

6. Lai CC, Shih TP, Ko WC, Tang HJ, Hsueh PR (2020) Severe acute respiratory syndrome coronavirus 2 (SARS-CoV-2) and coronavirus disease-2019 (COVID-19): The epidemic and the challenges. International Journal of Antimicrobial Agents 7: 4. [Crossref]

7. Jaimes JA, et al. Structural modelling of 2019-novel coronavirus (nCoV) spike protein reveals a proteolytically sensitive activation loop as a distinguishing feature compared to SARS-CoV and related SARS like coronaviruses. 
8. Lai CC, Liu YH, Wang CY, Wang YH, Hsueh SC, et al. (2020) Asymptomatic carrier state, acute respiratory disease, and pneumonia due to severe acute respiratory syndrome coronavirus 2 (SARS-CoV2): Facts and myths. Journal of Microbiology Immunology and Infection S1684-1182(20)30040-30042. [Crossref]

9. Coronavirus disease 2019 (COVID-19) Situation Repoer-43. World Health Organization.

10. Coronavirus: WHO declares global emergency. Politico

11. Coronavirus: Woman in 70s becomes first virus fatality in UK.

12. Ipplotio G, Hui DS, Ntoumi F, Maeurer M, Zumla A (2020) Toning down the 2019nCoV media hype- and restoring hope. Lancet 8: 230-231. [Crossref]

13. Jin YH, Cai L, Cheng ZS, Cheng H, Deng T, et al. (2020) A rapid advice guideline for the diagnosis and treatment of 2019 novel coronavirus (2019-nCoV) infected pneumonia. Military Medical Research 7: 4. [Crossref]

14. Rothan HA, Byrareddy SN (2020) The epidemiology and pathogenesis of coronavirus disease (COVID-19) outbreak. Journal of Autoimmunity. [Crossref]

15. Wang W, Tang J, Wei F (2020) Updated understanding of the outbreak of 2019 novel coronavirus (2019-nCoV) in Wuhan, China. J. Med. Virol 92; 441-447. [Crossref]

16. Yan X, Yu Y, Xu J, Shu H, Xia J, et al. (2020) Clinical course and outcomes of critically ill patients with SARS-CoV2 pnuemonia in Wuhan, china: a single centres, retrospective, observational study. The Lancet: Respiratory Medicine. [Crossref]

17. Chen N, Zhou M, Dong X, Qu J, Gong F, et al. (2020) Epidemiological and clinical characteristics of 99 cases of 2019 novel coronavirus pneumonia in Wuhan, China: a descriptive study. The Lancet 395: 507-513. [Crossref]

18. Guo L, Wei D, Zhang X, Wu Y, Li Q, et al. (2019) Clinical Features Predicting Mortality Risk in Patients with Viral Pneumonia: The MulBSTA score. Frontiers in Microbiology. [Crossref]

19. Vardavas CI, Nikitat K (2020) COVID-19 and smoking: A syetmatic review of the evidence. Tobacco Induced Diseases. [Crossref]

20. Alsharani MS, Sindi A, Alshamsi F, Al-Omari A, EI Tahan M, et al. (2018) Extracorporeal membrane oxygenation for severe middle east respiratory syndrome coronavirus. Ann Intensive Care 8: 3. [Crossref]

21. Pharm Tai, Combes A, Rozé H, Chevret S, Mercat A, et al. (2013) Extracorporeal Membrane Oxygenation for Pandemic Influenza A (H1N1) -inducted Acute Respiratory Distress. A cohort Study and Propensity-matched analysis. Am J Respir Crit Care Med 187: 376-285. [Crossref]

22. World Health Organization: Clinical management of severe acute respiratory infection when novel coronavirus (2019-nCoV) infection is suspected

23. Stockman LJ, Bellamy R, Gamer P (2006) SARS: systematic review of treatment effects. PLoS Med 3. [Crossref]

24. Sameed M, Meng Z, Ellen TM (2019) EOLIA trial: the future of extracorporeal membrane oxygenation in acute respiratory distress syndrome therapy? Breathe (Sheff) 15: 244-246. [Crossref]

25. Arabi Y, Mandourah Y, Al-Hameed F, Sindi AA, Almekhlafi GA, et al. (2018) Corticosteroid therapy for critically ill patients with middle east respiratory syndrome. Am J Respir Crit Care Med 197: 757-767. [Crossref]

26. Li YC, Bai WZ, Hashikawa T (2020) The neuroinvasive potential of SARS-CoV2 may play a role in the respiratory failure of COVID-19 patients. J Med Virol. [Crossref]

27. Propetchara E, Ketlay C, Palaga T (2020) Immune responses in COVID-19 and potential vaccines: Lessons learned from SARS and MERS epidemic. Asian Pacific Journal of Allergy and Immunology. [Crossref]

28. Xu X, Yu C, Qu J, Zhang L, Jiang S, et al. Imaging and clinical features of patients with 2019 novel coronavirus SARS-CoV2. European Journal of Nuclear Medicine and Molecular Imaging. [Crossref]

29. Ai T, Yang Z, Hou H, Zhan C, Chen C, et al. (2020) Correlation of Chest CT and RT-PCR testing in coronavirus disease 2019 (COVID-19) in China: A report of 1014 cases. Radiology. [Crossref]

30. Fang Y, Zhang H, Xie J, Lin M, Ying L, et al. Sensitivity of chest CT for covid-19: comparison to RT-PCR. Radiology. [Crossref]

31. Shen K, Yang Y, Wang T, Zhao D, Jiang Y, et al. (2020) Diagnosis, treatment and prevention of 2019 novel coronavirus infection in children: experts' consensus statement. World Journal of Paediatrics. [Crossref]

32. Hawes MK (2017) The Reappearance of Vaccine Preventable Diseases. VIGILINT.

33. Chen J (2020) Pathogenicity and transmissibility of 2019-nCoV - a quick overview and comparison with other emerging viruses. Microbes and Infection.

34. Chinazzi M, Davis JT, Ajelli M, Gioannini C, Litvinova M, et al. (2020) The effect of travel restrictions on the spread of the 2019 novel coronavirus (COVID-19) outbreak. Science. [Crossref]

35. Pasel A, Jernigan DB (2020) Initial public health response and interim clinical guidance for the 2019 novel coronavirus outbreak-unites states, December 31,
2019- February 4, 2020. Morbidity and Morality Weekly Report 69: 140-146. [Crossref]

36. Phan LT, Nguyen TV, Luong QC, Nguyen TV, Nguyen HT, et al. (2020) Importation and human-to-human transmission of a novel coronavirus in Vietnam. New England Journal of Medicine 282: 872-874. [Crossref]

37. Ong SWX, Tan YK, Chia PY, Lee TH, Ng OT, et al. (2020) Air surface environmental and personal protective equipment contamination by severe acute respiratory syndrome coronavirus 2 (SARS-CoV2) from a symptomatic treatment. JAMA. [Crossref]

38. New Coronavirus stable for hours on surfaces. National Institutes of Health

39. Chan LY, Leung CC, Lam TH, Cheng KK (2020) To wear or not to wear: WHO's confusing guidance on masks in the COVID-19 pandemic. BMJ.

40. Wang M, Cao R, Zhang L, Yang X, Liu J, et al. (2020) Remdesivir and chloroquine effectively inhibit the recently emerged novel coronavirus (2019-nCoV) in vitro. Cell Research 30: 269-271. [Crossref]

41. Li H, Zhou Y, Zhang M, Wang H, Zhao Q, et al. (2020) Updated approaches against SARS-CoV2. Antimicrob. Agents Chemother. [Crossref]

42. Gautret P, Lagier JC, Parola P, Hoang VT, Meddeb L, et al. (2020) Hydroxychloroquine and azithromycin as a treatment of COVID-19: results of an open-label non randomized clinical trial. Int J Antimicrob Agents.[Crossref]

43. Favalli EG, et al. (2020) COVID-19 infection and rheumatoid arthritis: Faraway, so close! Autoimmunitu Reviews.

44. Hoffman M, Kleine-Weber H, Schroeder S, Krüger N, Herrler T, et al. (2020) SARS-CoV2 cell entry depends on ACE2 and TMPRSS2 and is blocked by a clinically proven protease inhibitor. CELL. [Crossref]

45. Sommerstein $\mathrm{R}$, et al. Preventing a covid-19 pandemic. BMJ.

46. National Institute for Health and Care Excellence: Sunitinib.

47. Richardson P, Griffin I, Tucker C, Smith D, Oechsle O, et al. Baricitinib as potential treatment for 2019-nCoV acute respiratory disease. Lancet. [Crossref]

48. Guo YR, Cao QD, Hong ZS, Tan YY, Chen SD, et al. (2020) The origin, transmission and clinical therapies on coronavirus disease 2019 (COVID-19) outbreak- an update on the status. Miliatary Medical Research 7: 11. [Crossref]

\section{Citation:}

Worku E and Worku D (2020) SARS-Cov2: A review of this novel coronavirus. Internal Med Res Open J Volume 5(1): 1-6. 